\title{
Wong-Type Dermatomyositis: An Extremely Rare Disease in Childhood
}

\author{
Mario Diplomatico ${ }^{1}$, Orsola Ametrano ${ }^{2}$, Maria Elena Errico ${ }^{3}$, \\ Angela Mauro ${ }^{4}$, Roberto Rega ${ }^{5}$, Rita Sottile ${ }^{4}$
}

\author{
1 Department of Woman, Child and of General and Specialized Surgery, University of Campania, Naples, Italy \\ 2 Dermatology Unit, AORN Santobono-Pausilipon, Naples, Italy \\ 3 Pathology Unit, AORN Santobono-Pausilipon, Naples, Italy \\ 4 Rheumatology Unit, AORN Santobono-Pausilipon, Naples, Italy \\ 5 University of Naples Federico II, Italy
}

Key words: dermatomyositis, pediatric rheumatology, dermatology

Citation: Diplomatico M, Ametrano O, Errico ME, Mauro A, Rega R, Sottile R. Wong-type dermatomyositis: an extremely rare disease in childhood. Dermatol Pract Concept. 2019;9(4):325-326. DOI: https://doi.org/10.5826/dpc.0904a22

Accepted: July 3, 2019; Published: October 31, 2019

Copyright: (02019 Diplomatico et al. This is an open-access article distributed under the terms of the Creative Commons Attribution License, which permits unrestricted use, distribution, and reproduction in any medium, provided the original author and source are credited.

Funding: None.

Competing interests: The authors have no conflicts of interest to disclose.

Authorship: All authors have contributed significantly to this publication.

Corresponding author: Mario Diplomatico, Department of Woman, Child and of General and Specialized Surgery, University of Campania, Via Luigi De Crecchio 2, 80138, Napoli, Italy. Email: mario.diplomatico@gmail.com

\section{Introduction}

Some cutaneous manifestations can hide systemic symptoms of dermatomyositis (DM). The Wong-type dermatomyositis (W-DM) should be considered in the differential diagnosis in children with overlapping symptoms of DM and pityriasis rubra pilaris (PRP). This rare form of DM is reported in only 27 cases in the literature: 6 cases of pediatric age and only 3 with associated cutaneous features of PRP ( 2 females aged 1 and 18 years and a 12-year-old boy) [1]. Our patient was the fourth case of W-DM with associated cutaneous features of PRP and the first under the age of 10 years. Punch biopsy and knowledge of rare diseases improve the diagnostic ability and therapy. Differential diagnosis includes lupus erythematosus, PRP, and psoriasis.

We wish to draw attention to an extremely rare condition in children and, through the clinical images, we highlight the peculiarity of this rare form of DM.

\section{Case Presentation}

A 7-year-old boy was hospitalized because of asthenia, bilateral palpebral edema, and rash. Follicular and nonfollicular erythematous and hyperkeratotic papules were detected on his limbs, merging into red hyperkeratotic patches/plaques on the outer side of the legs (Figure 1, A and B). Purple plaques on the knees (Figure 1C), erythematous desquamating patches on the elbows (Figure 1D), and Gottron papules (Figure 2A) on metacarpophalangeal articulations were present. Blood tests showed increasing muscle enzyme levels. Elbow punch biopsy showed ortho/parakeratosis with perivascular lymphocytic infiltrate, necrotic keratinocytes, and vacuolar interface dermatitis; a dilated follicular infundibulum with keratotic plug was also found (Figure 2, B and C) [1,2]. W-DM was diagnosed and an MRI scan of his legs was compatible with DM. We decided to treat the patient with a combination of a high-dose short course of intravenous methylprednisolone at a dose of $30 \mathrm{mg} / \mathrm{kg} /$ day (maximum dose $1 \mathrm{~g}$ ) for 3 

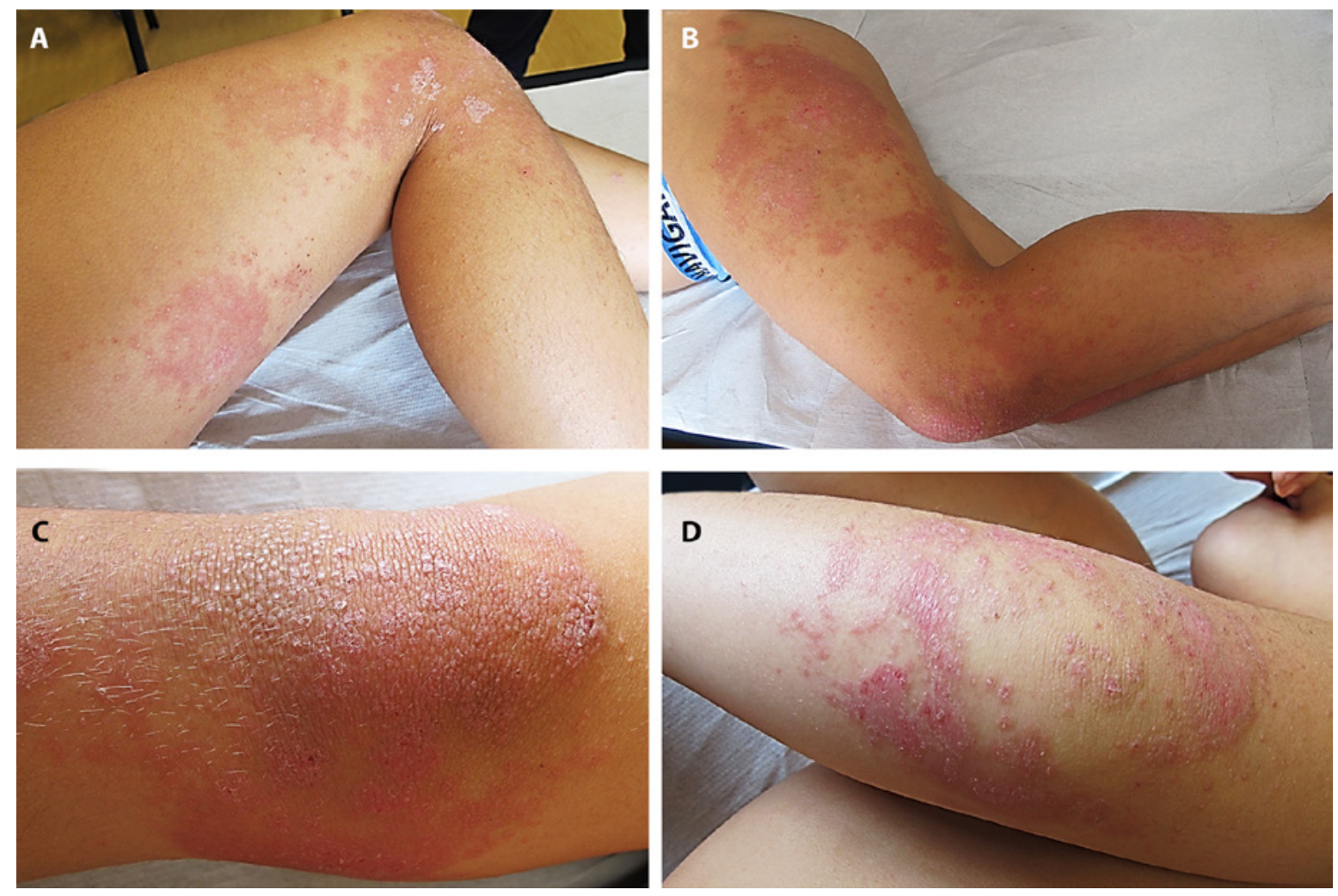

Figure 1. (A,B) Follicular and nonfollicular erythematous and hyperkeratotic papules merging into red hyperkeratotic patches/ plaques on the outer side of the legs. (C) Purple plaques with hyperkeratotic papules on the knees. (D) Erythematous desquamating patches on the elbows. [Copyright: @2019 Diplomatico et al.]
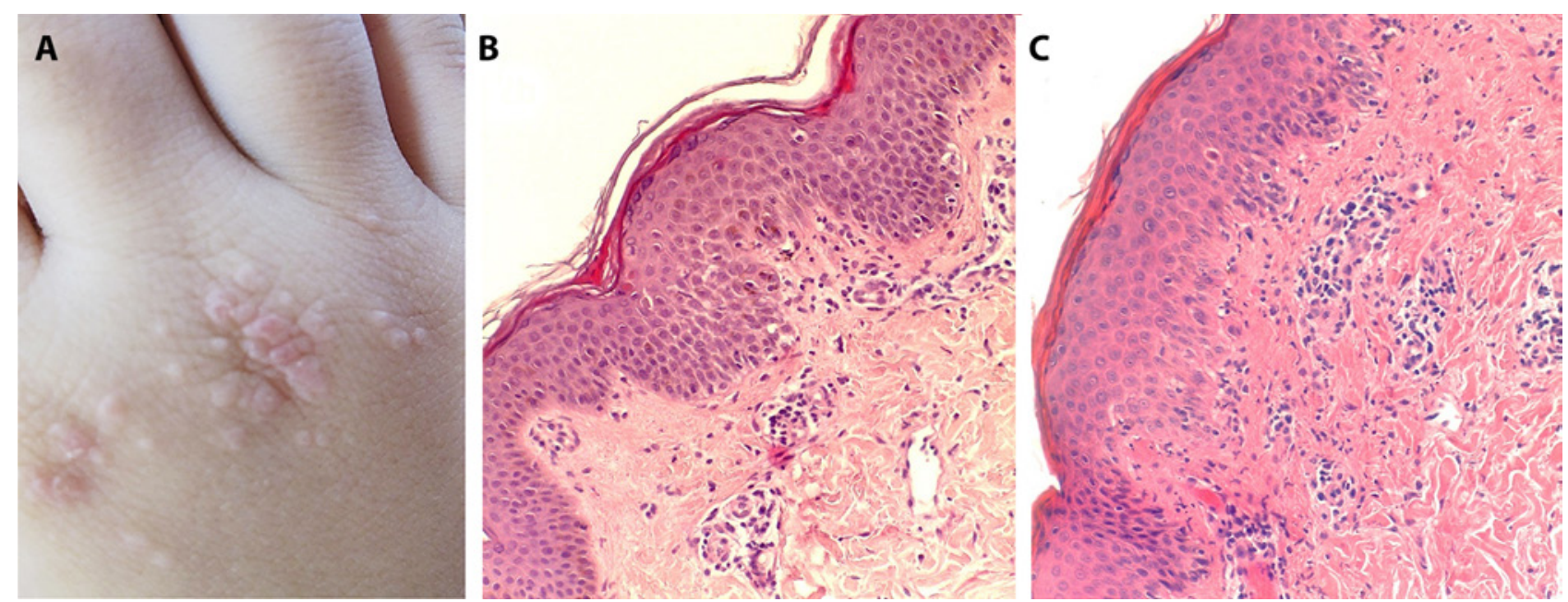

Figure 2. (A) Gottron papules. (B,C) Ortho/parakeratosis with perivascular lymphocytic infiltrate, necrotic keratinocytes, and vacuolar interface dermatitis; there is also a dilated follicular infundibulum with keratotic plug. [Copyright: @2019 Diplomatico et al.]

consecutive days and methotrexate $15 \mathrm{mg} / \mathrm{m}^{2}$ (maximum 25 $\mathrm{mg} / \mathrm{dose}$ once weekly, with associated folic acid $1 \mathrm{mg} /$ day), obtaining a dramatic improvement in his clinical condition.

\section{Conclusions}

W-DM should be considered in the differential diagnosis also in younger children with overlapping symptoms of DM and PRP [3].

\section{References}

1. Mutasim DF, Egesi A, Spicknall KE. Wong-type dermatomyositis: a mimic of many dermatoses. J Cutan Pathol. 2016;43(9):781-786.

2. Umanoff N, Fisher A, Carlson JA. Wong-type dermatomyositis showing porokeratosis-like changes (columnar dyskeratosis): a case report and review of the literature. Dermatopathology (Basel). 2015;2(1):1-8.

3. Canavan T, Sidorsky T, Doan LT, et al. A case of Wong-type dermatomyositis with concomitant anti-MDA5 features. J Am Acad Dermatol. 2014;70(3):e62-e64. 